\title{
Role of Perioperative Transesophageal Echocardiography in Left Ventricular Apical Pseudoaneurysm Surgery
}

\author{
${ }^{1}$ Imran H Bhat, ${ }^{2}$ Srinath Damodaran, ${ }^{3}$ Krishna P Gourav, ${ }^{4}$ Ganesh K Munirathinam, ${ }^{5}$ Sunder L Negi,
}

${ }^{6}$ Shyam KS Thingnam ${ }^{7}$ Goverdhan D Puri, ${ }^{8}$ Virendra K Arya

\begin{abstract}
Left ventricular apical pseudoaneurysm following myocardial infarction is a rare entity and often fatal due to rupture of free wall, thus warranting an urgent surgery. Although transthoracic echocardiography (TTE) plays a significant role in the diagnosis of pseudoaneurysm, transesophageal echocardiography (TEE) remains an indispensable tool during perioperative period. In this report, we are describing a case of left ventricular apical pseudoaneurysm planned for an urgent surgery.
\end{abstract}

Keywords: Cardiopulmonary bypass, Left ventricular apical pseudoaneurysm, Myocardial infarction, Transesophageal echocardiography.

How to cite this article: Bhat IH, Damodaran S, Gourav KP, Munirathinam GK, Negi SL, Thingnam S, Puri GD, Arya VK. Role of Perioperative Transesophageal Echocardiography in Left Ventricular Apical Pseudoaneurysm Surgery. J Perioper Echocardiogr 2018;6(1):20-24.

Source of support: Nil

Conflict of interest: None

\section{INTRODUCTION}

Left ventricular free wall rupture following myocardial infarction remains the most dreadful complication. The advancement of diagnostic technology has dramatically decreased mortality and morbidity due to myocardial infarction. ${ }^{1}$ Although left ventricular pseudoaneurysms are rare with the incidence of $0.1 \%$ following myocardial infarction, chance of rupture is more common due to lack of myocardial support, thus warranting urgent surgery. Current literature mentioned that 30 to $45 \%$ of left ventricular pseudoaneurysm has high chance for rupture. ${ }^{2-4}$ Difference between true aneurysm and pseudoaneurysm is given in Table 1. Transthoracic echocardiography and

\footnotetext{
${ }^{1-4}$ Senior Resident, ${ }^{5}$ Assistant Professor, ${ }^{6,7}$ Professor and Head ${ }^{8}$ Professor

1-5,7,8 Department of Anesthesia and Intensive Care, Postgraduate Institute of Medical Education and Research, Chandigarh, India

${ }^{6}$ Department of Cardiovascular and Thoracic Surgery, Postgraduate Institute of Medical Education and Research, Chandigarh, India

Corresponding Author: Srinath Damodaran, Senior Resident Department of Anesthesia and Intensive Care, Postgraduate Institute of Medical Education and Research, Chandigarh, India e-mail: drsrinath88@gmail.com
}

Table 1: Differences between true aneurysm and pseudoaneurysm

\begin{tabular}{ll}
\hline True aneurysm & Pseudoaneurysm \\
\hline 4-20\% incidence following & 0.1\% incidence following \\
myocardial infarction & myocardial infarction \\
Wide base & Narrow base \\
Thinned out myocardium & Ruptured myocardium \\
Noncontractile & Dyskinetic \\
Low risk of free rupture & High risk of free rupture \\
No adherent pericardium & Contained by pericardium \\
More common in anterior & More common in posterior \\
wall and apex & and lateral wall \\
\hline
\end{tabular}

cardiac magnetic resonance imaging (MRI) have been shown to be important in formulating preoperative surgical plan but have limited role during the intraoperative period. ${ }^{5}$ Two- (2D) and three-dimensional (3D) TEE remain superior to TTE in evaluating and delineating the pseudoaneurysm from true aneurysm. ${ }^{6}$ In this report, we present a rare case of apical left ventricular pseudoaneurysm who underwent successful repair.

\section{CASE REPORT}

A 52-year-old male with weight of $85 \mathrm{~kg}$ and height of $181 \mathrm{~cm}$ who had myocardial infarction 2 months back came to our hospital for a routine medical check-up. He had hypertension, type II diabetes mellitus, dyslipidemia, and gastroesophageal reflux. He was a known smoker and an alcoholic since 20 years. On examination, the pulse was regular with rate of $89 / \mathrm{min}$ and blood pressure was 128/78 $\mathrm{mm} \mathrm{Hg}$ in his right upper limb. Electrocardiogram showed Q wave in the II, V3, V4, and V5 leads, implying an old anterolateral wall infarction. Chest $X$-ray was normal. The TTE showed distorted apical bulging region with no evidence of pericardial collection, suggesting left ventricular aneurysm. Patient's left ventricular ejection fraction (LVEF) by modified Simpson's method was $45 \%$. Regional wall motion abnormality (RWMA) was observed in the left anterior descending (LAD) territory in para-sternal short axis view. Cardiac catheterization revealed $100 \%$ block in the LAD artery with normal right coronary artery and left circumflex arteries. Patient was planned for grafting of LAD artery and aneurysmal repair of left ventricle (LV). 
In order to allay the patient's anxiety, alprazolam $(0.25 \mathrm{mg})$ was given orally a night before planned surgery. In addition to all standard American Society of Anesthesiology monitors, TEE monitoring was planned. Anesthesia was induced with fentanyl $2 \mu \mathrm{g} / \mathrm{kg}$, ketamine $0.25 \mathrm{mg} / \mathrm{kg}$, and propofol $1 \mathrm{mg} / \mathrm{kg}$. Endotracheal intubation was facilitated with vecuronium $0.1 \mathrm{mg} / \mathrm{kg}$. TEE was performed with GE Health care Vivid E9 machine (Norway) after induction using 6VT probe. Surprisingly, TEE (2D and 3D) showed an apical pseudoaneurysm communicating with LV through narrow base formed by ruptured myocardium of left ventricular apex (Figs 1 to 4 ) which was not appreciated by TTE. According to Becker and van Mantgem ${ }^{7}$ classification, our patient had type III LV rupture. Regional wall motion abnormality in LAD region was reconfirmed by TEE in transgastric mid-papillary short axis view. In 17-segmental model, $13,14,15$, and 16 segments had dyskinesia in TEE. Left ventricular ejection fraction, by modified Simpson's method, was calculated to be $45 \%$ with no valvular

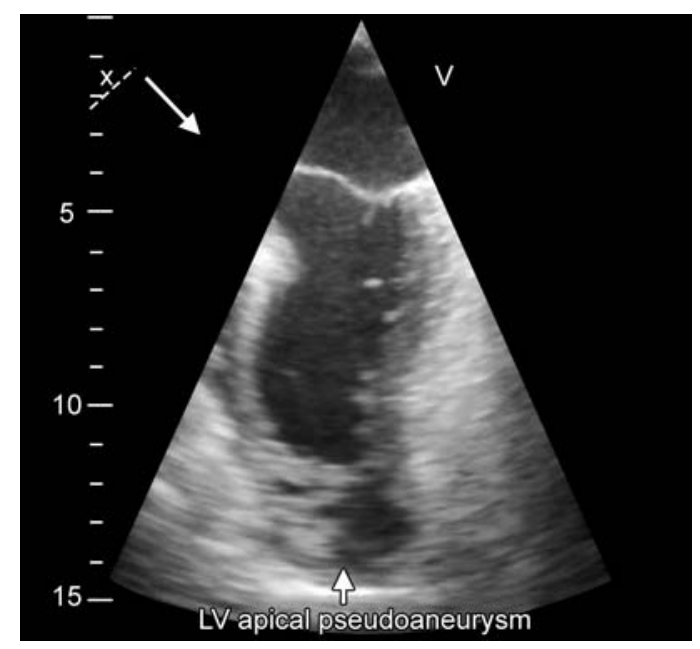

Fig. 1: Mid-esophageal four-chamber view showing left ventricular apical pseudoaneurysm

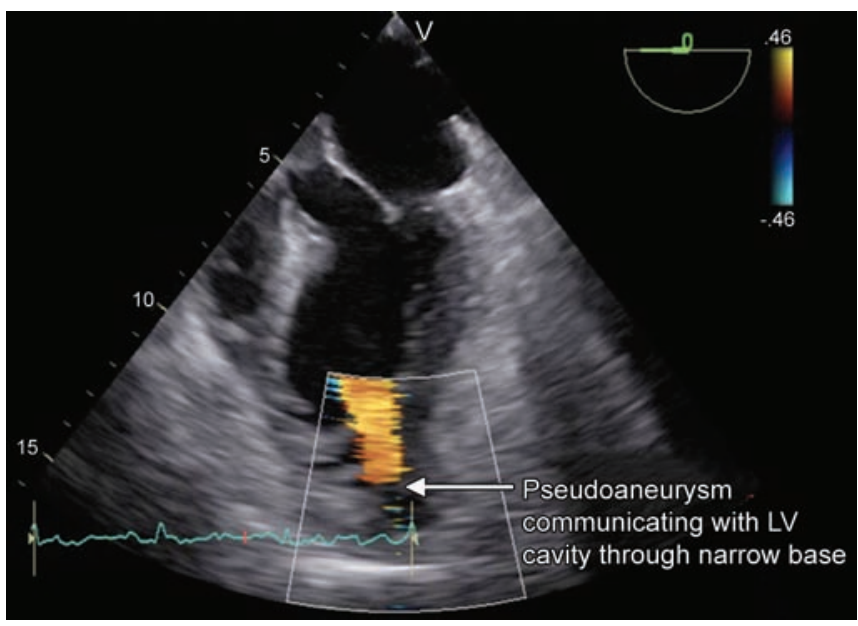

Fig. 3: Mid-esophageal four-chamber view with color Doppler showing communication of apical pseudoaneurysm with LV regurgitation correlated with TTE. A grade III atheroma in the arch and descending thoracic aorta and a grade II atheroma in the ascending aorta were also observed in TEE. Hence, surgery plan was changed to Dor procedure and grafting of LAD.

Femoral vessels were exposed and cannulated before sternotomy after adequate anticoagulation in order to establish extracorporeal circulation in case any untoward incident happens during sternotomy and pericardial dissection. After midline sternotomy, pericardium was opened carefully, leaving the apical adherent pericardium intact. The femoral cannulae were clamped. Cardiopulmonary bypass $(\mathrm{CPB})$ was established using aortic and dual-stage venous cannulation. The pseudoaneurysm was dissected following cardiac arrest and excluded from left ventricular cavity (Figs 5 and 6) and the neck region was closed with pericardium. Thrombotic and necrotic tissue, present in the aneurysmal region, was excised. Grafting in the LAD region was abandoned because of poor target vessel. Aortic cross clamp was

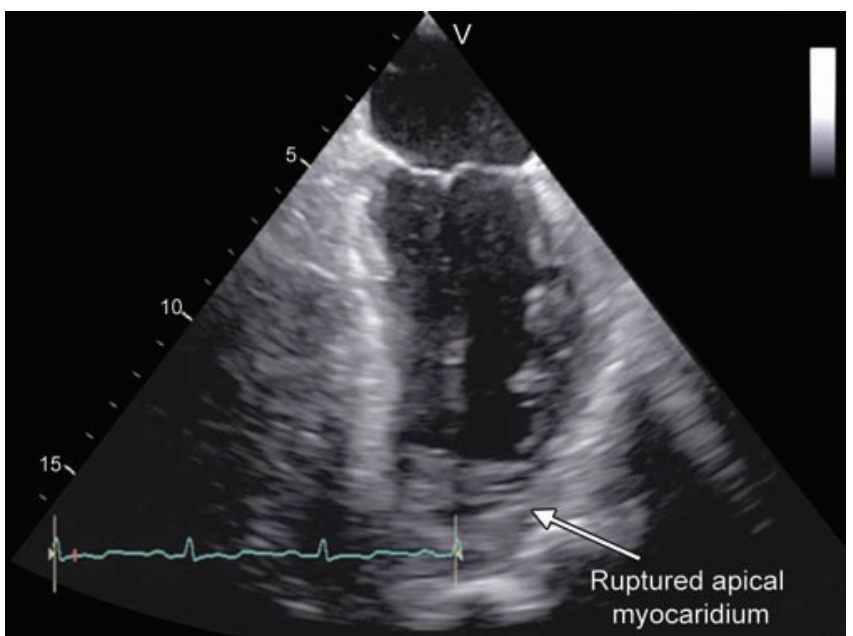

Fig. 2: Mid-esophageal two-chamber view showing left ventricular apical pseudoaneurysm

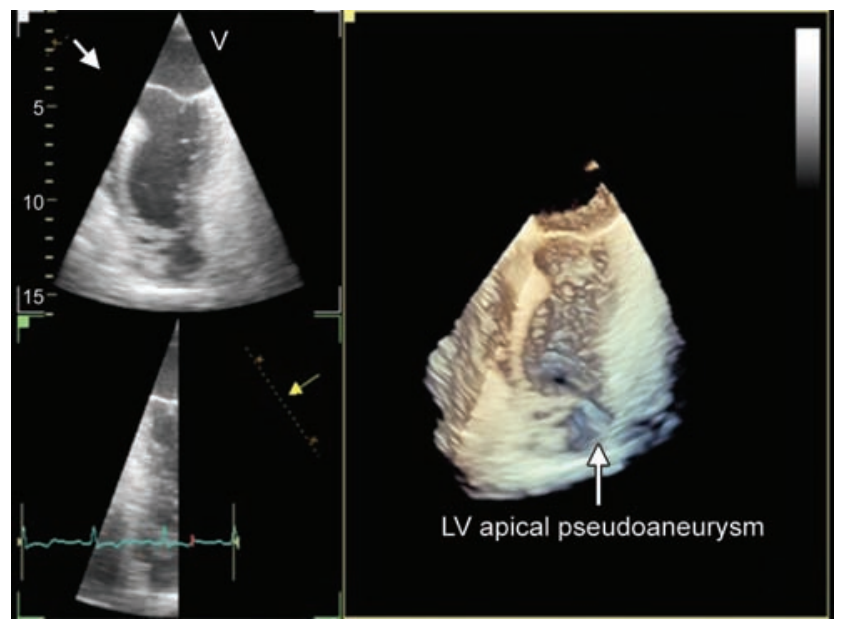

Fig. 4: Three-dimensional image showing left ventricular apical pseudoaneurysm 


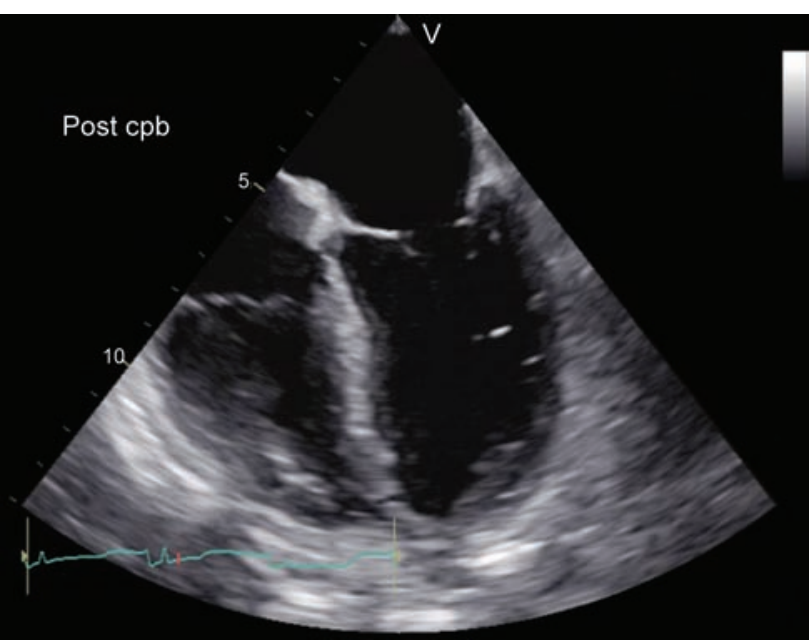

Fig. 5: Mid-esophageal four-chamber view after surgical repair of left ventricular apical pseudoaneurysm

removed after 48 minutes. The patient was successfully weaned off from $\mathrm{CPB}$ using milrinone (loading dose of $50 \mu \mathrm{g} / \mathrm{kg}$ on CPB followed by infusion at the rate of $0.3 \mu \mathrm{g} / \mathrm{kg} / \mathrm{min}$ ). Post-CPB TEE revealed an LVEF of 50\% and trivial mitral regurgitation with adequate repair of aneurysmal area. The improved LVEF may be attributed to inotropic use and exclusion of necrotic area. The RWMA persisted in the LAD region in post-CPB period. Patient was shifted to surgical intensive care unit and extubated after 5 hours of mechanical ventilation. Postoperative pain was controlled with morphine infusion. After uneventful postoperative period, patient was shifted to ward on postoperative day 3 and discharged from hospital on postoperative day 5 .

\section{DISCUSSION}

Left ventricular apical pseudoaneurysm contained by pericardium is a rare complication following acute myocardial infarction. The pseudoaneurysm following transmural infarction has high mortality of up to $20 \%{ }^{8}$ Other etiologies for pseudoaneurysm include cardiac surgery, trauma, and infection. ${ }^{9,10}$ Pseudoaneurysm is usually associated with pericarditis which results in visceral and parietal pericardial adhesion, thus preventing development of hemopericardium and resultant cardiac tamponade. The pseudoaneurysmal wall contains dense fibrous tissue that lacks myocardial fibers and coronary vessels and has a narrow communication between the aneurysmal and ventricular cavity. A true aneurysm, in contrast, results in focal convex deformation of the heart, has wide communication between the aneurysmal and ventricular cavity, contains myocardial fibers, and is lined by the endothelium. ${ }^{11}$ In our case, LV was communicating with pseudoaneurysm via a narrow neck formed by the ruptured apical myocardium as visualized in TEE.

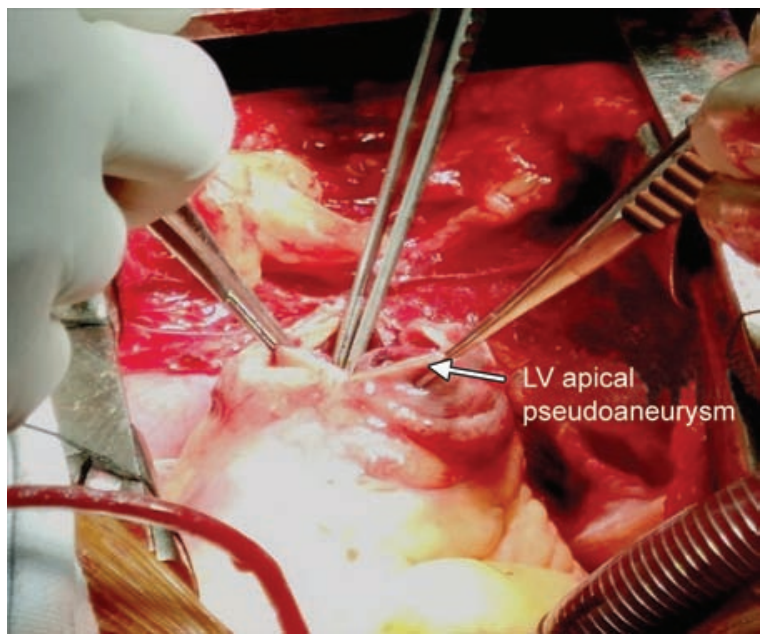

Fig. 6: Surgical repair of left ventricular apical pseudoaneurysm

Clinical presentation of pseudoaneurysm widely varies, which ranges from asymptomatic to sudden cardiac death, which signifies the necessity of rapid and prompt intervention soon after diagnosis is made. Untreated pseudoaneurysm can cause arrhythmias, embolism, congestive cardiac failure, and even sudden death due to massive hemopericardium and cardiac tamponade. ${ }^{9,12}$

Elderly patients, female sex, 6th decade of life, preexisting systemic hypertension, absence of previous episode of angina pectoris or infarction, symptoms of pericarditis, ST-T wave changes, peak values of creatine kinase-MB fraction more than $150 \mathrm{IU} / \mathrm{L}$, absence of a mural thrombus, absence of collateral vessels in the area of infarction, and therapy in the form of thrombolysis given more than 7 hours after the onset of chest pain are the risk factors for free wall rupture..$^{13}$ Our patient was in his 6th decade of life, smoker, hypertensive, and had absent flow in LAD with no collateral vessels. Hence, our patient had high risk for developing an aneurysm rupture.

Multimodal cardiac imaging is necessary in accurate and definitive delineation of pseudoaneurysm, which helps in perioperative management. In our case, TTE is able to identify the LV aneurysmal region, but distinguishing true aneurysm from pseudoaneurysm warrants detailed assessment. Poor window associated with obesity, chronic obstructive pulmonary disease, smoker, and suboptimal positioning cause difficulty in assessing the pseudoaneurysm, especially in the apical region with TTE. ${ }^{14}$ Although left ventricular apex lies near to the field of TTE window, TEE clarified the diagnosis in our case due to poor TTE window, as our patient was a smoker and obese. Furthermore, TEE helped in clearly delineating the exact location of the pseudoaneurysm and communication of the pseudoaneurysm with left ventricular cavity in the perioperative period to guide the surgeon, which was not clearly visualized in 
TTE. Use of high-frequency transducer than TTE and focused imaging on the region of interest in TEE helps in locating the mural thrombus, which is common in pseudoaneurysm of the LV. Examination with TEE further clarifies the function of $\mathrm{LV}$, involvement of mitral valves, and associated ventricular septal rupture and its location. Atherosclerotic plaques in the aorta is common in coronary artery disease patients, in which TEE is far more superior to TTE in identifying the exact location of atheroma, thus guiding the surgeon in aortic cannulation. Real-time monitoring by TEE throughout surgery helps in assessing cardiac function and maintaining hemodynamic stability during manipulation of heart. The 3D TEE technology further improves the visualization of pseudoaneurysm by delineating the dimensions, location, and communication with LV cavity. The 3D echocardiography is more useful in its presurgical assessment and for estimating diastolic volume evaluation of pseudoaneurysm in left ventricle. ${ }^{15}$ The TEE is also superior to TTE in identifying the thrombus within the pseudoaneurysm, and thus helps in avoiding excessive manipulation of heart during pre-CPB period. ${ }^{5}$

Intraoperative epicardial echocardiography with high-frequency probe provides optimal window for anterior structures like aorta, aortic valve, pulmonary valve, and pulmonary arteries. ${ }^{16}$ However, difficulty in obtaining two-chamber view for assessing left ventricular apical region and interruption of surgery for epicardial echocardiography are the major limitations in consistently using this technique in left ventricular pseudoaneurysm surgery.

Cardiac MRI, a noninvasive modality, is a valuable tool which provides valuable details of the pseudoaneurysm with its 3D technology, which aids in the surgical management. Lack of real-time assessment with cardiac MRI during intraoperative period limits its role in perioperative set-up, which makes TEE more reliable and helpful. ${ }^{17}$ The most accurate diagnostic method of diagnosing left ventricular pseudoaneurysm is left ventriculography. The major limitation of this technique is its invasive nature and use of contrast agents. ${ }^{9}$

Optimal management of pseudoaneurysm remains controversial because of wide spectrum of clinical presentations. Emergency surgery is required in patients with cardiogenic shock. Patients with stable hemodynamic need a planned but urgent surgery. ${ }^{18,19}$

\section{CONCLUSION}

Transesophageal echocardiography remains a valuable diagnostic modality in diagnosing and distinguishing pseudoaneurysm from true aneurysm, especially in the perioperative period. The ability in assessing ventricular function, identifying valvular pathologies and thrombus during intraoperative period makes TEE an indispensable tool in perioperative management of pseudoaneurysm surgery.

\section{REFERENCES}

1. Sirois JG. Acute myocardial infarction. Emerg Med Clin North Am 1995;13(4):759-769.

2. Alapati L, Chitwood WR, Cahill J, Mehra S, Movahed A. Left ventricular pseudoaneurysm: a case report and review of the literature. World J Clin Cases 2014 Apr;2(4):90-93.

3. Zoffoli G, Mangino D, Venturini A, Terrini A, Asta A, Zanchettin C, Polesel E. Diagnosing left ventricular aneurysm from pseudo-aneurysm: a case report and a review in literature. J Cardiothorac Surg 2009 Feb;4:11.

4. Komeda M, David TE. Surgical treatment of postinfarction false aneurysm of the left ventricle. J Thorac Cardiovasc Surg 1993 Dec;106(6):1189-1191.

5. Burns CA, Paulsen W, Arrowood JA, Tolman DE, Rose B, Fabian JA, Spratt JA. Improved identification of posterior left ventricular pseudo-aneurysms by transesophageal echocardiography. Am Heart J 1992 Sep;124(3):796-799.

6. Esakof DD, Vannan MA, Pandian NG, Cao QL, Schwartz SL, Bojar RM. Visualization of left ventricular pseudoaneurysm with panoramic transesophageal echocardiography. J Am Soc Echocardiogr 1994 Mar-Apr;7(2):174-178.

7. Becker AE, van Mantgem JP. Cardiac tamponade. A study of 50 hearts. Eur J Cardiol 1975 Dec;3(4):349-358.

8. Becker RC, Gore JM, Lambrew C, Weaver WD, Rubison RM, French WJ, Tiefenbrunn AJ, Bowlby LJ, Rogers WJ. A composite view of cardiac rupture in the United States National Registry of Myocardial Infarction. J Am Coll Cardiol 1996 May;27(6):1321-1326.

9. Frances C, Romero A, Grady D. Left ventricular pseudoaneurysm. J Am Coll Cardiol 1998 Sep;32(3):557-561.

10. Pretre R, Linka A, Jenni R, Turina MI. Surgical treatment of acquired left ventricular pseudoaneurysm. Ann Thorac Surg 2000 Aug;70(2):53-57.

11. Roberts WC, Morrow AG. Pseudoaneurysm of the left ventricle. An unusual sequel of myocardial infarction and rupture of the heart. Am J Med 1967 Oct;43(4):639-644.

12. Rogers JH, De Oliveira NC, Damiano RJ Jr, Rogers JG. Left ventricular apical pseudoaneurysm. Circulation 2002 Feb;105(8):e51-e52.

13. Hung MJ, Wang CH, Cherng WJ. Unruptured left ventricular pseudoaneurysm following myocardial infarction. Heart 1998 Jul;80(1):94-97.

14. Chirillo F, Cavarzerani A, Ius $\mathrm{P}$, Totis $\mathrm{O}$, Bruni A, Valfrè $C$, Stritoni P. Role of transthoracic, transesophageal, and transgastric two-dimensional and colour Doppler echocardiography in the evaluation of mechanical complications of acute myocardial infarction. Am J Cardiol 1995 Oct;76(11): 833-836.

15. Cacciapuoti F, Tirelli P, Cacciapuoti F. Left ventricular postinfarction pseudoaneurysm: diagnostic advantages of three-dimensional echocardiography. J Cardiovasc Echogr 2017 Apr-Jun;27(2):74-76.

16. Reeves ST, Glas KE, Eltzschig H, Mathew JP, Rubenson DS, Hartman GS, Shernan SK; American Society of Echocardiography; Society of Cardiovascular Anesthesiologists. Guidelines for performing a comprehensive epicardial 
echocardiography examination: recommendations of the American Society of Echocardiography and the Society of Cardiovascular Anesthesiologists. J Am Soc Echocardiogr 2007 Apr;20(4):427-437.

17. Kerber RE, Pesek-Bird C. Could cardiac magnetic resonance imaging replace cardiac ultrasound? Challenges from the echocardiography laboratory. J Cardiovasc Magn Reson 2002;4(2):289-290.
18. Maltais S, Ibrahim R, Basmadjian AJ, Carrier M, Bouchard D, Cartier R, Demers P, Ladouceur M, Pellerin M, Perrault LP. Postinfarction ventricular septal defects: towards a new treatment algorithm? Ann Thorac Surg 2009 Mar;87(3):687-692.

19. Daggett WM, Guyton RA, Mundth ED, Buckley MJ, McEnany MT, Gold HK, Leinbach RC, Austen WG. Surgery for post-myocardial infarct ventricular septal defect. Ann Surg 1977 Sep;186(3):260-271. 